\title{
Dynamics of a Nonautonomous Plant Disease Model with General Nonlinear Incidence Rate and Time-Varying Impulse
}

\author{
Jianping Wang1*, Xue Zhang², Juanjuan Wang1, Quanben Sun' \\ ${ }^{1}$ The Department of Basic Course Education, Ji'an College, Ji'an, China \\ ${ }^{2}$ College of Mathematics and Computer Science, Gannan Normal University, Ganzhou, China \\ Email: *jpw math@1 26.com
}

How to cite this paper: Wang, J.P., Zhang, X., Wang, J.J. and Sun, Q.B. (2019) Dynamics of a Nonautonomous Plant Disease Model with General Nonlinear Incidence Rate and Time-Varying Impulse. Journal of Applied Mathematics and Physics, 7, 2518-2530.

https://doi.org/10.4236/jamp.2019.710171

Received: September 17, 2019

Accepted: October 26, 2019

Published: October 29, 2019

Copyright $\odot 2019$ by author(s) and Scientific Research Publishing Inc. This work is licensed under the Creative Commons Attribution International License (CC BY 4.0).

http://creativecommons.org/licenses/by/4.0/ (c) (i) Open Access

\begin{abstract}
In this paper, for controlling the spread of plant diseases, a nonautonomous SEIS (Susceptible $\rightarrow$ Exposed $\rightarrow$ Infectious $\rightarrow$ Susceptible) epidemic model with a general nonlinear incidence rate and time-varying impulsive control strategy is proposed and investigated. This novel model could result in an objective criterion on how to control plant disease transmission by replanting of healthy plants and removal of infected plants. Using the method of small amplitude perturbation, the sufficient conditions under which guarantee the globally attractive of the disease-free periodic solution and the permanence of the disease are obtained, that is, the disease dies out if $R_{1}<1$, otherwise, the disease persists if $R_{2}>1$.
\end{abstract}

\section{Keywords}

Plant Disease, Nonautonomous Model, Time-Varying Impulse, General Nonlinear Incidence, Permanence

\section{Introduction}

Plant viruses or pathogens are an important constraint to crop production worldwide, and cause serious losses in agriculture and forestry. For example, Huanglongbing (HLB) has no cure and affects all citrus varieties, reducing the productivity of orchards because the fruits of infected plants have poor quality and, in extreme cases, infection leads to plant death [1] [2] [3]. Several plant diseases caused by plant viruses in cassava (Manihot esculenta) and sweet potato (Ipomoea batatas) are among the main constraints to sustainable production of these vegetatively propagated staple food crops in lesser-developed countries [4] [5] 
[6]. Epidemics of many polycyclic plant diseases caused by ascomycete fungal pathogens are invited by wind-borne ascospores transported into the crop in autumn [7] and subsequently develop further through cycles of splash dispersed conidia [8]. Integrated disease management (IDM) has been developed gradually by famers to reduce the numbers of infected individuals to a tolerable level and aims to minimize losses and maximize returns which combine biological, cultural, and chemical tactics and so on. A cultural control strategy including replanting, and/or removing diseased plants is a widely accepted treatment for plant epidemics which involves periodic inspections followed by removal of the detected infected plants [9]-[17]. Therefore, we have turned more attention to plant diseases.

Mathematical models play an important role in understanding the epidemiology of plant diseases. Applications of mathematical approach to plant epidemics were reviewed by Van der Plank and Kranz. There are many authors establishing mathematical models to describe the transmission of plant disease. Meng and $\mathrm{Li}$ [6] have investigated vegetatively propagated plant diseases and developed a mathematical model with impulsive cultural control strategies as follows:

$$
\left\{\begin{array}{l}
\frac{\mathrm{d} S(t)}{\mathrm{d} t}=-\frac{\beta S(t) I(t)}{1+\alpha S(t)}-\mu S(t)+\omega I(t), \\
\frac{\mathrm{d} I(t)}{\mathrm{d} t}=\frac{\beta S(t) I(t)}{1+\alpha S(t)}-(d+\omega) I(t), \\
S\left(t^{+}\right)=S(t)+\rho, \\
I\left(t^{+}\right)=(1-r) I(t),
\end{array}\right\} t=n \tau .
$$

Zhang and Meng take into account time delays in modeling equations and the models take the form of delay differential equations [18]. The reasonable time delay plant disease models are established as follows:

$$
\left\{\begin{array}{l}
\frac{\mathrm{d} S(t)}{\mathrm{d} t}=-\beta \mathrm{e}^{-\mu \tau} \frac{S(t) I(t)}{1+\alpha S(t)}-\mu S(t)+\omega I(t), \\
\frac{\mathrm{d} I(t)}{\mathrm{d} t}=\beta \mathrm{e}^{-\mu \tau} \frac{S(t) I(t)}{1+\alpha S(t)}-(d+\omega) I(t), \\
S\left(t^{+}\right)=S(t)+\rho, \\
I\left(t^{+}\right)=(1-r) I(t),
\end{array}\right\} t=n \tau .
$$

The above epidemic models discussed with constant coefficients and saturation incidence rate. However, the nonautonomous phenomenon is prevalent in the real life, which should be more realistic than autonomous systems. For example, the climate changes may lead to the variation of the disease spreading, seasonal cultivation of the plant, and so on. More recently, many authors pointed out that a nonlinear incidence rate may be more realistic during the disease transmission process. For example, Teng and Zhang [19] have proposed an SIS epidemic 
model with generalized incidence rate $g(I(t)) S(t)$. To address the time-varying nature of the coefficients more realistically, nonautonomous model with general nonlinear incidence rate and time-varying impulse has been introduced.

The remainder of the paper is organized as follows. In Section 2, we formulate the impulsive epidemic model and also simplify the original system (3). In Section 3, we introduce some useful lemmas and the basic reproduction number of the model. In Sections 4 and 5, we proved the global stability of the disease-free equilibrium and permanence of the model, respectively. In the finally section, we present numerical simulations that demonstrate the theoretical results and give some control and prevention measures.

\section{Model Formulation and Preliminary}

In this section, the plant population is subdivided into three groups: susceptible plants $S$, latent infected plants $E$ and infected plants $I$. We assume that replanting susceptible plants and roguing latent infected and infected plants at the same time. Motivated by the above factors, we propose a non-autonomous epidemic model incorporated general nonlinear incidence rate and time-varying impulse as follows:

$$
\left\{\begin{array}{l}
\frac{\mathrm{d} S(t)}{\mathrm{d} t}=-f(t, S, E)-g(t, S, I)-\mu(t) S(t)+v(t) I(t), \\
\frac{\mathrm{d} E(t)}{\mathrm{d} t}=f(t, S, E)+g(t, S, I)-\mu(t) E(t)-\sigma(t) E(t), \\
\frac{\mathrm{d} I(t)}{\mathrm{d} t}=\sigma(t) E(t)-\mu(t) I(t)-v(t) I(t), \\
S\left(t^{+}\right)=S(t)+\delta_{k}, \\
E\left(t^{+}\right)=\left(1-p_{k}\right) E(t), \\
I\left(t^{+}\right)=\left(1-\theta_{k}\right) I(t),
\end{array}\right\} t=t_{k}, k \in N,
$$

The model is satisfied with the following assumptions.

(H1) $S(t), E(t), I(t)$ denote the number of susceptible, latent and infected plants, respectively. The initial conditions are $S\left(t_{0}\right)>0, I\left(t_{0}\right)>0$ and $R\left(t_{0}\right)>0$.

(H2) $S(t), E(t)$ and $I(t)$ are left continuous for $\left[t_{0},+\infty\right)$.

(H3) $\mu(t), v(t)$ are the nature death, recovery rate at time $t$, respectively. $\sigma(t)$ is the rate at which latent population becomes infective population at time $t$.

(H4) The coefficients $\mu(t), v(t)$ and $\sigma(t)$ are assumed to be nonnegative, continuous and bounded $\omega$-periodic functions in the interval $\left[t_{0},+\infty\right)$.

(H5) $t_{k}$ represent pulse time. There exists a positive integer $q$ such that $t_{k+q}=t_{k}+\omega$ for all $k \in N$.

(H6) $\delta_{k}, p_{k}$ and $\theta_{k}\left(0 \leq \delta_{k}, p_{k}, \theta_{k} \leq 1\right)$ are the pulse replanting rates of susceptible plants, roguing rates of latent plants and infected plants at each fix 
time $t=t_{k}$, respectively, and $\delta_{k}=\delta_{q+k}, \quad p_{k}=p_{q+k}, \quad \theta_{k}=\theta_{q+k}$ for all $k \in N$.

(H7) The general nonlinear incidence rates $f(t, S, E)$ and $g(t, S, I)$ are piecewise continuous, nonnegative, periodic functions with period $\omega$. The form of $f(t, S, E)$ and $g(t, S, I)$ are as follows:

$$
f(t, S, E)=\left\{\begin{array}{c}
f_{1}(t, S, E), \quad t \in\left(t_{0}+n \omega, t_{1}+n \omega\right], \\
\vdots \\
f_{q}(t, S, E), t \in\left(t_{q-1}+n \omega, t_{q}+n \omega\right],
\end{array}\right.
$$

and

$$
g(t, S, I)=\left\{\begin{array}{cc}
g_{1}(t, S, I), & t \in\left(t_{0}+n \omega, t_{1}+n \omega\right], \\
\vdots \\
g_{q}(t, S, I), & t \in\left(t_{q-1}+n \omega, t_{q}+n \omega\right],
\end{array}\right.
$$

for all nonnegative integer $n$, and $f_{i}(t, 0, E)=f_{i}(t, S, 0)=0$, $g_{i}(t, 0, I)=g_{i}(t, S, 0)=0$ for $i=1,2, \cdots, q$.

Before analysis, we introduce some notations and definitions. Let $C$ denote the space of all bounded continuous functions. Given $f \in C$, we let

$$
f^{M}=\sup _{t \geq 0} f(t), f^{L}=\inf _{t \geq 0} f(t) .
$$

If $f$ is $\omega$-periodic, then the average value of $f$ on a time interval $[0, \omega]$ can be defined as

$$
\bar{f}=\frac{1}{\omega} \int_{0}^{\omega} f(t) \mathrm{d} t .
$$

Lemma 1. Consider the following nonautonomous impulsive differential equations:

$$
\left\{\begin{array}{l}
\frac{\mathrm{d} S(t)}{\mathrm{d} t}=-\mu(t) S(t), t \neq t_{k}, k \in N, \\
S\left(t^{+}\right)=S(t)+\delta_{k}, t=t_{k}, k \in N,
\end{array}\right.
$$

has a unique positive $\omega$-periodic solution $S^{*}(t)$ which is globally asymptotically stable.

Proof. Integrating and solving the first equation of system (4) between pulses for $n \omega+t_{k-1}<t \leq n \omega+t_{k}, k=1,2, \cdots, q, k \in N$.

$$
S(t)=S\left(\left(n \omega+t_{k-1}\right)^{+}\right) \exp \left(-\int_{n \omega+t_{k-1}}^{t} \mu(s) \mathrm{d} s\right)
$$

where $S\left(\left(n \omega+t_{k-1}\right)^{+}\right)=S\left(n \omega+t_{k-1}\right)+\delta_{k}$, and $S\left(n \omega+t_{k-1}\right)$ be the initial value at time $n \omega+t_{k-1}$.

It follows from above equation and using the second equation of system (4), we get

$$
S\left(\left(n \omega+t_{1}\right)^{+}\right)=S\left(\left(n \omega+t_{0}\right)^{+}\right) \exp \left[-\int_{n \omega+t_{0}}^{n \omega+t_{1}} \mu(s) \mathrm{d} s\right]+\delta_{1},
$$

and 


$$
\begin{aligned}
S\left(\left(n \omega+t_{2}\right)^{+}\right)= & S\left(\left(n \omega+t_{0}\right)^{+}\right) \exp \left[-\int_{n \omega+t_{0}}^{n \omega+t_{2}} \mu(s) \mathrm{d} s\right] \\
& +\delta_{1} \exp \left[-\int_{n \omega+t_{1}}^{n \omega+t_{2}} \mu(s) \mathrm{d} s\right]+\delta_{2} .
\end{aligned}
$$

Obviously, $S\left(\left(n \omega+t_{q}\right)^{+}\right)=S\left(\left((n+1) \omega+t_{0}\right)^{+}\right)$, using the inductive method, namely that starts with the observations and theories are proposed towards the end of the research process as a result of observation, we have

$$
\begin{aligned}
S\left(\left(n \omega+t_{q}\right)^{+}\right)= & S\left(\left(n \omega+t_{0}\right)^{+}\right) \exp \left[-\int_{n \omega+t_{0}}^{n \omega+t_{q}} \mu(s) \mathrm{d} s\right] \\
& +\sum_{k=1}^{q} \delta_{k} \exp \left[-\int_{n \omega+t_{k}}^{n \omega+t_{q}} \mu(s) \mathrm{d} s\right] .
\end{aligned}
$$

Set $U_{n}=S\left(n \omega+t_{0}\right)$. From (5) and $t_{q}-t_{0}=\omega$, we have

$$
U_{n+1}=U_{n} \exp \left[-\int_{n \omega+t_{0}}^{n \omega+t_{q}} \mu(s) \mathrm{d} s\right]+\sum_{k=1}^{q} \delta_{k} \exp \left[-\int_{n \omega+t_{k}}^{n \omega+t_{q}} \mu(s) \mathrm{d} s\right] \triangleq f\left(U_{n}\right) .
$$

$f$ is the stroboscopic map. It is easy to see that system (6) has unique positive equilibrium:

$$
\begin{aligned}
S_{0}^{*}=\frac{\sum_{k=1}^{q} \delta_{k} \exp \left[-\int_{n \omega+t_{k}}^{n \omega+t_{q}} \mu(s) \mathrm{d} s\right]}{1-\exp \left[-\int_{n \omega+t_{0}}^{n \omega+t_{q}} \mu(s) \mathrm{d} s\right]}, \\
S^{*}(t)=\left[S_{0}^{*} \exp \left(-\int_{n \omega+t_{0}}^{n \omega+t_{k}} \mu(s) \mathrm{d} s\right)+\sum_{k=1}^{q} \delta_{k} \exp \left(-\int_{n \omega+t_{k}}^{n \omega+t_{q}} \mu(s) \mathrm{d} s\right)\right] \\
\times \exp \left[-\int_{n \omega+t_{k-1}}^{t} \mu(s) \mathrm{d} s\right] .
\end{aligned}
$$

It's easy to see that system (3) has a unique disease-free periodic solution $\left(S^{*}(t), 0,0\right)$.

\section{Global Attractivity of the Disease-Free Periodic Solution}

$0.6 \mathrm{~cm}$ Before discuss the attractivity of the disease-free periodic solution of system (3), we firstly make the following assumption:

A): There exist two positive, continuous, periodic functions $\xi_{i}(t), \beta_{i}(t)$ with the period $\omega$, that is $\xi_{i}(t)=\xi_{i}(t+\omega), \quad \beta_{i}(t)=\beta_{i}(t+\omega)$, for all $i=1,2, \cdots, q$, such that $g_{i}(t, S, I) \leq \xi_{i}(t) S(t) I(t), f_{i}(t, S, E) \leq \beta_{i}(t) S(t) E(t)$, for $t \geq t_{0}$.

Theorem 1. If $R_{1}<1$ and system (3) satisfies the assumption (A), then the disease-free periodic solution $\left(S^{*}(t), 0,0\right)$ is globally attractive, where

$$
R_{1}=\frac{\sum_{i=1}^{q} \int_{t_{i-1}}^{t_{i}}\left(\alpha_{i}(t) S^{*}(t)\right) \mathrm{d} t}{\sum_{i=1}^{q}\left[\int_{t_{i-1}}^{t_{i}} \mu(t) \mathrm{d} t-\ln \left(1-a_{i}\right)\right]},
$$

and

$$
a_{i}=\min \left\{p_{i}, \theta_{i}\right\}, \alpha_{i}=\max \left\{\xi_{i}(t), \beta_{i}(t)\right\}, i=1,2, \cdots, q .
$$

Proof. Let $(S(t), E(t), I(t))$ be any solution of system (3). Since $R_{1}<1$, 
there exists a sufficiently small number $\epsilon_{1}>0$ such that

$$
\Omega_{1} \triangleq \exp \left\{\sum_{i=1}^{q} \int_{t_{i-1}}^{t_{i}}\left[\alpha_{i}(\tau)\left(S^{*}(\tau)+\epsilon_{1}\right)-\mu(\tau)\right] \mathrm{d} \tau+\sum_{i=1}^{q} \ln \left(1-a_{i}\right)\right\}<1 .
$$

By the comparison theorem [20], we can get that there exists a positive constant $t^{1}\left(>t_{0}\right)$ such that

$$
S(t)<S^{*}(t)+\epsilon_{1}
$$

for all $t \geq t^{1}$. It follows from second, third equations of system (3) and (10), we have that, for $t \in\left(n \omega+t_{i-1}, n \omega+t_{i}\right](i=1,2, \cdots, q)$ and $t \geq t^{1}$,

$$
\begin{aligned}
& \frac{\mathrm{d} E(t)}{\mathrm{d} t}+\frac{\mathrm{d} I(t)}{\mathrm{d} t} \\
& =f(t, S, E)+g(t, S, I)-\mu(t)(E(t)+I(t))-v(t) I(t) \\
& \leq \beta_{i}(t) S(t) E(t)+\xi_{i}(t) S(t) I(t)-\mu(t)(E(t)+I(t)) \\
& \leq \alpha_{i}(t)\left(S^{*}(t)+\epsilon_{1}\right)(E(t)+I(t))-\mu(t)(E(t)+I(t)) \\
& =\alpha_{i}(t)\left(S^{*}(t)+\epsilon_{1}-\mu(t)\right)(E(t)+I(t)),
\end{aligned}
$$

where $\alpha_{i}(t)$ is the same to (8). Thus, we get that

$$
\begin{aligned}
E(t)+I(t) \leq & \left(E\left(\left(t_{i-1}+n \omega\right)^{+}\right)+I\left(\left(t_{i-1}+n \omega\right)^{+}\right)\right) \\
& \times \exp \int_{t_{i-1}+n \omega}^{t}\left[\alpha_{i}(t)\left(S^{*}(t)+\epsilon_{1}-\mu(t)\right)\right] \mathrm{d} t \\
= & {\left[\left(1-p_{i-1}\right) E\left(t_{i-1}+n \omega\right)+\left(1-\theta_{i-1}\right) I\left(t_{i-1}+n \omega\right)\right] } \\
& \times \exp \int_{t_{i-1}+n \omega}^{t}\left[\alpha_{i}\left(S^{*}(t)+\epsilon_{1}\right)-\mu(t)\right] \mathrm{d} t \\
\leq & \left(1-a_{i-1}\right)\left(E\left(t_{i-1}+n \omega\right)+I\left(t_{i-1}+n \omega\right)\right) \\
& \times \exp \int_{t_{i-1}+n \omega}^{t}\left[\alpha_{i}(t)\left(S^{*}(t)+\epsilon_{1}\right)-\mu(t)\right] \mathrm{d} t .
\end{aligned}
$$

By using the similar method, we can deduce from (11) that for

$$
\begin{aligned}
t \in\left(t_{i-1}+n \omega, t_{i}+n \omega\right] & =\left(1-a_{i-1}\right)\left(E\left(n \omega+t_{i-1}\right)+I\left(n \omega+t_{i-1}\right)\right) \\
& \times \exp \int_{n \omega+t_{i-1}}^{t}\left[\alpha_{i}(\tau)\left(S^{*}(\tau)+\epsilon_{1}\right)-\mu(\tau)\right] \mathrm{d} \tau \\
\leq & \prod_{j=1}^{i-1}\left(1-a_{j}\right)\left(E\left(n \omega+t_{i-1}\right)+I\left(n \omega+t_{i-1}\right)\right) \\
& \times \exp \left\{\int_{n \omega+t_{0}}^{n \omega+t_{1}}\left[\alpha_{1}(\tau)\left(S^{*}(\tau)+\epsilon_{1}\right)-\mu(\tau)\right] \mathrm{d} \tau+\cdots\right. \\
& \left.+\int_{n \omega+t_{i-1}}^{t}\left[\alpha_{i}(\tau)\left(S^{*}(\tau)+\epsilon_{1}\right)-\mu(\tau)\right] \mathrm{d} \tau\right\} \\
= & \prod_{j=1}^{i-1}\left(1-a_{j}\right)\left(E\left(n \omega+t_{i-1}\right)+I\left(n \omega+t_{i-1}\right)\right) \\
& \times \exp \left\{\sum_{j=1}^{i} \int_{n \omega+t_{j-1}}^{n \omega+t_{j}}\left[\alpha_{i}(t)\left(S^{*}(\tau)+\epsilon_{1}\right)-\mu(\tau)\right] \mathrm{d} \tau\right\} .
\end{aligned}
$$

Especially, when $t=(n+1) \omega+t_{0}$, we have 


$$
\begin{aligned}
& E\left((n+1) \omega+t_{0}\right)+I\left((n+1) \omega+t_{0}\right) \\
= & I\left(n \omega+t_{q}\right)+E\left(n \omega+t_{q}\right) \\
\leq & \left(1-a_{q}\right)\left(E\left(n \omega+t_{q}\right)+I\left(n \omega+t_{q}\right)\right) \\
\leq & \prod_{i=1}^{q}\left(1-a_{i}\right)\left[E\left(n \omega+t_{0}\right)+I\left(n \omega+t_{0}\right)\right] \\
& \times \exp \left\{\sum_{i=1}^{q} \int_{t_{i-1}}^{t_{i}}\left[\alpha_{i}(\tau)\left(S^{*}(\tau)+\epsilon_{1}\right)-\mu(\tau)\right] \mathrm{d} \tau\right\} \\
= & {\left[E\left(n \omega+t_{0}\right)+I\left(n \omega+t_{0}\right)\right] } \\
& \times \exp \left[\sum_{i=1}^{q} \int_{t_{i-1}}^{t_{i}}\left[\alpha_{i}(\tau)\left(S^{*}(\tau)+\epsilon_{1}\right)-\mu(\tau)\right] \mathrm{d} \tau+\sum_{i=1}^{q} \ln \left(1-a_{i}\right)\right] \\
= & \Omega_{1}\left[E\left(n \omega+t_{0}\right)+I\left(n \omega+t_{0}\right)\right] .
\end{aligned}
$$

Therefor, for any positive integer $s$, we get that

$$
\begin{aligned}
& E\left(\left((n+s) \omega+t_{0}\right)^{+}\right)+I\left(\left((n+s) \omega+t_{0}\right)^{+}\right) \\
\leq & \Omega^{s}\left[E\left(\left(n \omega+t_{0}\right)^{+}\right)+I\left(\left(n \omega+t_{0}\right)^{+}\right)\right] .
\end{aligned}
$$

Combining with (9), we have

$$
E\left(\left((n+s) \omega+t_{0}\right)^{+}\right)+I\left(\left((n+s) \omega+t_{0}\right)^{+}\right) \rightarrow 0, \text { as } s \rightarrow+\infty .
$$

According to (12) and (13), we know that

$$
\lim _{t \rightarrow+\infty}(E(t)+I(t))=0 \text {. }
$$

For above mentioned $\epsilon_{1}$, there exists $t^{2}\left(>t^{1}\right)$, we get $E(t)+I(t)<\epsilon_{1}$ for all $t>t^{2}$.

From Lemma 1 and (14), we can see that the disease-free periodic solution $\left(S^{*}(t), 0,0\right)$ is global attractive.

\section{Permanence of the Disease}

In this section, we mainly obtain the sufficient conditions for the permanence of system (3). We give the following assumption at first:

(B) There exist two positive, continuous, periodic functions $\gamma_{i}(t), \eta_{i}(t)$ with period $\omega$, such that $f_{i}(t, S, E) \geq \gamma_{i}(t) S(t) I(t), g_{i}(t, S, I) \geq \eta_{i}(t) S(t) I(t)$, for $t \geq t_{0}$.

Denote $S^{* *}(t)$ by the solution of the following system:

$$
\left\{\begin{array}{l}
\frac{\mathrm{d} Z(t)}{\mathrm{d} t}=-\left(\alpha_{i}(t) \epsilon_{2}+\mu(t)\right) Z(t), t \neq t_{k}, k \in N, \\
Z\left(t^{+}\right)=Z(t)+\delta_{k}, t=t_{k}, k \in N .
\end{array}\right.
$$

According to Lemma 1, we can obtain that the system has a unique positive $\omega$-periodic solution $S^{* *}(t)$ which is globally asymptotically stable.

Theorem 2. If $R_{2}>1$ and system (3) satisfies the Hypotheses (A) and (B), the system (3) is permanent, where

$$
R_{2}=\frac{\sum_{i=1}^{q} \int_{t_{i-1}}^{t_{i}} \varphi_{i}(t) S^{* *}(t) \mathrm{d} t}{\sum_{i=1}^{q}\left[\int_{t_{i-1}}^{t_{i}}(\mu(t)+v(t)) \mathrm{d} t-\ln \left(\left(1-b_{i}\right)\right]\right.} .
$$


Proof: Since $R_{2}>1$, it's easily to see that there exists a sufficiently small $\epsilon_{2}>0$, such that

$$
\Omega_{2} \triangleq \prod_{i=1}^{q}\left(1-b_{i}\right) \exp \left\{\sum_{i=1}^{q} \int_{t_{i-1}}^{t_{i}}\left[\varphi_{i}(\tau)\left(S^{* *}(\tau)-\epsilon_{2}\right)-(\mu(t)+v(\tau))\right] \mathrm{d} \tau\right\}>1 .
$$

In order to illustrate the conclusion, we first prove the disease is uniformly weakly persistent, that is, there exists a positive constant $m>0$, such that $\limsup _{t \rightarrow+\infty}(E(t)+I(t)) \geq m$. By contradiction, for above given $\epsilon_{2}>0$, there exists a constant $t^{3}>0$, such that $E(t)+I(t)<\epsilon_{2}$, for all $t>t^{3}$.

According to assumption (A) and the first equation of system (3), we know

$$
\begin{aligned}
\frac{\mathrm{d} S(t)}{\mathrm{d} t} & =-f(t, S, E)-g(t, S, I)-\mu(t) S(t)+v(t) I(t) \\
& \geq-\beta_{i}(t) S(t) E(t)-\xi_{i}(t) S(t) I(t)-\mu(t) S(t) \\
& \geq-\alpha_{i}(t) \epsilon_{2} S(t)-\mu(t) S(t) \\
& =-\left(\alpha_{i}(t) \epsilon_{2}+\mu(t)\right) S(t) .
\end{aligned}
$$

Then we have

$$
\left\{\begin{array}{l}
\frac{\mathrm{d} S(t)}{\mathrm{d} t} \geq-\left(\alpha_{i}(t) \epsilon_{2}+\mu(t)\right) S(t), t \neq t_{k}, k \in N, \\
S\left(t^{+}\right)=S(t)+\delta_{k}, t=t_{k}, k \in N .
\end{array}\right.
$$

By comparison theorem, we have $S(t) \geq Z(t)$ and $Z(t) \rightarrow S^{* *}(t)$ as $t \rightarrow+\infty$, where $Z(t)$ is the solution of the following comparison system:

$$
\left\{\begin{array}{l}
\frac{\mathrm{d} Z(t)}{\mathrm{d} t}=-\left(\alpha_{i}(t) \epsilon_{2}+\mu(t)\right) Z(t), t \neq t_{k}, k \in N, \\
Z\left(t^{+}\right)=Z(t)+\delta_{k}, t=t_{k}, k \in N .
\end{array}\right.
$$

Therefore, for above mentioned $\epsilon_{2}$, there exists an integer $n^{*}>0$, such that

$$
S(t) \geq Z(t) \geq S^{* *}(t)-\epsilon_{2},
$$

for all $t>t^{3}+n^{*} \omega$

For above mentioned $t^{3}+n^{*} \omega$, we can get that there exists a positive integer $N_{1}$, such that $N_{1} \omega \geq t^{3}+n^{*} \omega$. Hence for all $n \omega+t_{i-1}<t<n \omega+t_{i},\left(n \geq N_{1}, i=1,2, \cdots, q\right)$, by the second and third equations of system (3) and (17), we have

$$
\begin{aligned}
& \frac{\mathrm{d} E(t)}{\mathrm{d} t}+\frac{\mathrm{d} I(t)}{\mathrm{d} t} \\
& =f(t, S, E)+g(t, S, I)-\mu(t)(E(t)+I(t))-v(t) I(t) \\
& \geq \gamma_{i}(t) S(t) E(t)+\eta_{i} S(t) I(t)-\mu(t)(E(t)+I(t))-v(t) I(t) \\
& \geq \varphi_{i}(t) S(t)(E(t)+I(t))-\mu(t)(E(t)+I(t))-v(t)(E(t)+I(t)) \\
& \geq\left[\varphi_{i}(t)\left(S^{* *}-\epsilon_{2}\right)-\mu(t)-v(t)\right](E(t)+I(t)),
\end{aligned}
$$

where $\varphi_{i}(t)=\min \left\{\gamma_{i}(t), \eta_{i}(t)\right\},(i=1,2, \cdots, q)$ are defined. In addition, in view of system (3), we yeild 


$$
E\left(t^{+}\right)+I\left(t^{+}\right)=\left(1-p_{i}\right) E(t)+\left(1-\theta_{i}\right) I(t) \geq\left(1-b_{i}\right)(E(t)+I(t)),
$$

where $b_{i}=\max \left\{p_{i}, \theta_{i}\right\},(i=1,2, \cdots, q)$ can be seen.

Then, we consider impulsive comparison system:

$$
\left\{\begin{array}{l}
\frac{\mathrm{d} y_{2}(t)}{\mathrm{d} t}=\left[\varphi_{i}(t)\left(S^{* *}(t)-\epsilon_{2}\right)-\mu(t)-v(t)\right] y_{2}(t), t \neq t_{k}, k \in N, \\
y_{2}\left(t^{+}\right)=\left(1-b_{k}\right) y_{2}(t), t=t_{k}, k \in N . \\
y_{2}\left(t_{0}^{+}\right)=E_{0}+I_{0}>0 .
\end{array}\right.
$$

By solving above impulsive differential equation, we can obtain that for

$$
\begin{aligned}
n \omega+t_{i-1}< & t<n \omega+t_{i},\left(n \geq N_{1}\right), i=1,2, \cdots, q, \\
y_{2}(t)= & \prod_{j=1}^{i-1}\left(1-b_{j}\right) y_{2}\left(\left(n \omega+t_{0}\right)^{+}\right) \exp \left[\int_{n \omega+t_{0}}^{n \omega+t_{1}} \varphi_{i}(\tau)\left(S^{* *}(\tau)-\epsilon_{2}\right) \mathrm{d} \tau+\cdots\right. \\
& \left.+\int_{n \omega+t_{i-1}}^{t} \varphi_{i}(\tau)\left(S^{* *}(\tau)-\epsilon_{2}\right) \mathrm{d} \tau\right] \cdot \exp \left[\int_{n \omega+t_{0}}^{n \omega+t_{1}}-(\mu(\tau)+v(\tau)) \mathrm{d} \tau+\cdots\right. \\
& \left.+\int_{n \omega+t_{i-1}}^{n \omega+t}-(\mu(\tau)+v(\tau)) \mathrm{d} \tau\right] .
\end{aligned}
$$

Furthermore, when $t=(n+1) \omega+t_{0}$, we have

$$
\begin{aligned}
& y_{2}\left(((n+1) \omega)^{+}+t_{0}\right)=\left(1-b_{j}\right) y_{2}\left(n \omega+t_{q}\right) \\
& =\prod_{i=1}^{q}\left(1-b_{i}\right) y_{2}\left(n \omega+t_{0}\right) \exp \left[\sum_{i=1}^{q} \int_{t_{i-1}}^{t_{i}} \varphi_{i}(\tau)\left(S^{* *}(\tau)-\epsilon_{2}\right) \mathrm{d} \tau\right] \\
& \cdot \exp \left[\sum_{i=1}^{q} \int_{t_{i-1}}^{t_{i}}-(\mu(\tau)+v(\tau)) \mathrm{d} \tau\right] \\
& =y_{2}\left((n \omega)^{+}+t_{0}\right) \exp \left\{\sum_{i=1}^{q} \int_{t_{i-1}}^{t_{i}}\left[\varphi_{i}(\tau)\left(S^{* *}(\tau)-\epsilon_{2}\right)-(\mu(\tau)+v(\tau))\right] \mathrm{d} \tau\right. \\
& \left.+\sum_{i=1}^{q} \ln \left(1-b_{i}\right)\right\} \\
& =\Omega_{2} y_{2}\left((n \omega)^{+}+t_{0}\right)
\end{aligned}
$$

Therefore, for any positive integer $l_{2}$, we have $y_{2}\left(\left(n+l_{2}\right) \omega+t_{0}\right) \geq \Omega_{2}^{l_{2}} y_{2}\left(n \omega+t_{0}\right)$. It follows from (19) that

$$
y_{2}\left(\left(n+l_{2}\right) \omega+t_{0}\right) \geq \Omega_{2}^{l_{2}} y_{2}\left(n \omega+t_{0}\right) \rightarrow+\infty,
$$

as $l_{2} \rightarrow+\infty$. By the comparison theorem, we have $\lim _{t \rightarrow+\infty}(E(t)+I(t))=+\infty$, which is a contradiction to $0<E(t)+I(t)<\epsilon_{2}$. Thus the claim is proved.

By the claim, we are left to consider the following two possibilities:

Case 1. $E(t)+I(t)>\epsilon_{2}$ for all large $t$.

Case 2. $E(t)+I(t)$ oscillates about $\epsilon_{2}$ for all large $t$.

The first case implies that the result holds. Then we will consider the second possibility. At first, set $\underline{t}>t^{3}$ and $\bar{t}>t^{3}$ be large enough such that

$E(\underline{t})+I(\underline{t}) \geq \epsilon_{2}, E(\bar{t})+I(\bar{t})=\epsilon_{2}$. and $E(t)+I(t)<\epsilon_{2}$, for $t \in(\underline{t}, \bar{t})$.

There are two possible subcases for $\underline{t}$.

Case (I). If $\underline{t}=t_{i}+n \omega$ ( $n$ is a nonnegative integer and $i=1,2, \cdots, q$ ), then $E(\underline{t})+I(\underline{t})>\epsilon_{2}$ and $\left(1-b_{i}\right) \epsilon_{2}<\left(1-b_{i}\right)(E(\underline{t})+I(\underline{t})) \leq E\left(\underline{t}^{+}\right)+I\left(\underline{t}^{+}\right)<\epsilon_{2}$, where $b_{i}=\max \left\{p_{i}, \theta_{i}\right\},(i=1,2, \cdots, q)$. We claim that there must exist a positive 
constant $m$, such that $E(t)+I(t) \geq m$, for $t \in(\underline{t}, \bar{t})$. Then, we will consider two possibilities in terms of the size of $\underline{t}$ and $\bar{t}$.

i) If $\bar{t}-\underline{t} \leq n^{*} \omega$, where $n^{*}$ is defined in (17), then from system (3), we have

$$
\left\{\begin{aligned}
& \frac{\mathrm{d} E(t)}{\mathrm{d} t}+\frac{\mathrm{d} I(t)}{\mathrm{d} t}=f(t, S, E)+g(t, S, I)-\mu(t)(E(t)+I(t))-v(t) \\
& \geq-\left(\mu^{M}+v^{M}\right)(E(t)+I(t)), t \neq t_{k}, k \in N, \\
& E\left(t^{+}\right)+I\left(t^{+}\right) \geq\left(1-b_{k}\right)(E(t)+I(t)), t=t_{k}, k \in N .
\end{aligned}\right.
$$

From (20), we have

$$
\begin{aligned}
& E(t)+I(t) \\
\geq & {\left[\prod_{i=1}^{q}\left(1-b_{i}\right)\right]^{n^{*}+1}(E(t)+I(t)) \exp \left[-\left(\mu^{M}+v^{M}\right)(t-\underline{t})\right] } \\
\geq & {\left[\prod_{i=1}^{q}\left(1-b_{i}\right)\right]^{n^{*}+1} \epsilon_{2} \exp \left[-\left(\mu^{M}+v^{M}\right) n^{*} \omega\right] } \\
\triangleq & m,
\end{aligned}
$$

for all $t \in[\underline{t}, \bar{t}]$.

ii) If $\bar{t}-\underline{t} \geq n^{*} \omega$, in view of the discussion in (i), we have $E(t)+I(t) \geq m$, for all $t \in\left[\underline{t}, \underline{t}+n^{*} \omega\right]$. Next, we show that $E(t)+I(t) \geq m$ for all $t \in\left(\underline{t}+n^{*} \omega, \underline{t}\right]$.

Otherwise, there exists a constant $t^{*}>0$ such that

$$
E(t)+I(t) \geq m,
$$

for all $t \in\left[\underline{t}, \underline{t}+t^{*}+n^{*} \omega\right) . \quad E\left(\underline{t}+t^{*}+n^{*} \omega\right)+I\left(\underline{t}+t^{*}+n^{*} \omega\right) \geq m$ and $E(t)+I(t)<m$, for all $0<t-\left(\underline{t}+t^{*}+n^{*} \omega\right) \ll 1$.

Next, we discuss two possibilities separately:

(a) For all $k \in n, \underline{t}+t^{*}+n^{*} \omega \neq t_{k}$.

It's easy to see system (18) holds on $\left[\underline{t}+n^{*} \omega, \bar{t}\right]$. So we can choose a proper $\rho>0$, such that $E\left(\underline{t}+n^{*} \omega+t^{*}\right)+I\left(\underline{t}+n^{*} \omega+t^{*}\right) \geq \rho\left(E_{0}+I_{0}\right) \geq m$.

By the comparison theorem, we have $0<t-\left(\underline{t}+n^{*} \omega+t^{*}\right) \ll 1$.

$$
\begin{aligned}
& E(t)+I(t) \\
& \geq\left(E\left(\underline{t}+n^{*} \omega+t^{*}\right)+I\left(\underline{t}+n^{*} \omega+t^{*}\right)\right) \\
& \quad \times \exp \int_{\underline{t}+n^{*} \omega+t^{*}}^{t}\left[\varphi_{i}(\tau)\left(S^{* *}(\tau)-\epsilon_{2}\right)-\mu(\tau)-v(\tau)\right] \mathrm{d} \tau \\
& \geq \rho\left(E_{0}+I_{0}\right) \exp \int_{\underline{t}+n^{*} \omega+t^{*}}^{t}\left[\varphi_{i}(\tau)\left(S^{* * *}(\tau)-\epsilon_{2}\right)-\mu(\tau)-v(\tau)\right] \mathrm{d} \tau
\end{aligned}
$$

In addition, (16) implies that

$$
\exp \int_{\underline{t}+n^{*} \omega+t^{*}}^{t}\left[\varphi_{i}(\tau)\left(S^{* *}(\tau)-\epsilon_{2}\right)-\mu(\tau)-v(\tau)\right] \mathrm{d} \tau \geq 1 .
$$

Then, we obtain that

$$
\begin{aligned}
& E(t)+I(t) \\
& \geq \rho\left(E_{0}+I_{0}\right) \exp \int_{\underline{\underline{t}+n^{*} \omega+t^{*}}}^{t}\left[\varphi_{i}(\tau)\left(S^{* * *}(\tau)-\epsilon_{2}\right)-\mu(\tau)-v(\tau)\right] \mathrm{d} \tau \\
& \geq \rho\left(E_{0}+I_{0}\right) \geq m .
\end{aligned}
$$


Then, $E(t)+I(t) \geq m$, for $0<t-\left(\underline{t}+n^{*} \omega+t^{*}\right) \ll 1$, which is a contradiction.

Therefore, $E(t)+I(t) \geq m$ for any $t \in[\underline{t}, \bar{t}]$.

(b) There exists a $k \in N$ such that $\underline{t}+n^{*} \omega+t^{*}=t_{k}$. The proof of (b) is similar to (a), so we omit it. Subcase (II). If for all $k \in N, \underline{t} \neq t_{k}$, then $E(\underline{t})+I(\underline{t})=\epsilon_{2}$. Using the same methods of subcase (I), we can easily get a positive constant $m$, such that $E(t)+I(t) \geq m$, for all $t \in[\underline{t}, \bar{t}]$.

Thus, we see that $E(t)+I(t) \geq m$ for any $t \in[\underline{t}, \bar{t}]$. Since this kind of interval $[\underline{t}, \bar{t}]$ is chosen in an arbitrary way, we conclude that $E(t)+I(t) \geq m$ for all large $t$.

According to our above discussion, the choice of $m$ is independent of the positive solution of system (3), and we have proved that any solution of system (3) satisfies $E(t)+I(t) \geq m$ for sufficiently large $t$, that is $\liminf _{t \rightarrow+\infty}(E(t)+I(t)) \geq m$. It is easy to obtain that, there exist positive constants $S_{*}$ such that $\liminf _{t \rightarrow+\infty} S(t) \geq S_{*}$. Therefore, the permanence of system (3) is proved.

\section{Conclusion}

In this paper, we have constructed a nonautonomous SEIS epidemic model with general nonlinear incidence and time-varying pulse control. On the basis of Theorems 1 and 2, we know that $R_{1}$ and $R_{2}$ are the threshold condition under the disease and become permanent or not. We have proved that the infected plants die out and the disease-free periodic solution is globally asymptotically attractive when the hypothesis (A) and $R_{1}<1$ hold. What's more, the infected plants persist when the hypotheses (A) and (B) hold and $R_{2}>1$.

\section{Acknowledgements}

The research has been supported by the Science and Technology Plan Projects of Jiangxi Provincial Education Department (GJJ151491, GJJ181359), Guidance Project of Ji'an Science and Technology Bureau, the Natural Science Foundation of Ji'an College (16JY103).

\section{Conflicts of Interest}

The authors declare no conflicts of interest regarding the publication of this paper.

\section{References}

[1] Bové, J.M. (2006) Huanglongbing: A Destructive, Newly Emerging, Century-Old Disease of Citrus. Journal of Plant Pathology, 88, 7-37.

[2] Vilamiu, R.G.A. and Ternes, S. (2012) A Model for Huanglongbing Spread between Citrus Plants Including Delay Times and Human Intervention. AIP Conference Proceedings, 1479, 2315. https://doi.org/10.1063/1.4756657

[3] Wang, J.P., Gao, S.J., et al. (2014) Threshold Dynamics of a Huanglongbing Model 
with Logistic Growth in Periodic Environments. Abstract and Applied Analysis, 2014, Article ID: 841367. https://doi.org/10.1155/2014/841367

[4] Gibson, R.W., Aritua, V., Byamukama, E., Mpembe, I. and Kayongo, J. (2004) Control Strategies for Sweet Potato Virus Disease in Africa. Virus Research, 100, 115-122. https://doi.org/10.1016/j.virusres.2003.12.023

[5] Thresh, J.M. and Cooter, R.J. (2005) Strategies for Controlling Cassava Mosaic Disease in Africa. Plant Pathology, 54, 587-614. https://doi.org/10.1111/j.1365-3059.2005.01282.x

[6] Meng, X.Z. and Li, Z.Q. (2010) The Dynamics of Plant Disease Models with Continuous and Impulsive Cultural Control Strategies. Journal of Theoretical Biology, 266, 29-40. https://doi.org/10.1016/j.jtbi.2010.05.033

[7] Fitt, I. and Evans, T. (1999) Ascospores as Primary Inoculum for Epidemics of White Leaf Spot (Mycosphaerella capsellae) in Winter Oilseed Rape in the UK. Plant Pathology, 48, 308-319. https://doi.org/10.1046/j.1365-3059.1999.00350.x

[8] Evenhuis, A., Verdam, B. and Zadoks, J.C. (1997) Splash Dispersal of Conidia of Mycocentrospora acerina in the Field. Plant Pathology, 46, 459-469.

https://doi.org/10.1046/j.1365-3059.1997.d01-42.x

[9] Jeger, M.J., Holt, J., van den Bosch, F. and Madden, L.V. (2004) Epidemiology of Insect-Transmitted Plant Viruses: Modelling Disease Dynamics and Control Interventions. Physiological Entomology, 29, 291-304.

https://doi.org/10.1111/j.0307-6962.2004.00394.x

[10] Zhang, X.S. and Holt, J. (2004) Mathematical Models of Cross Protection in the Epidemiology of Plant-Virus Diseases. Phytopathology, 91, 924-934. https://doi.org/10.1094/PHYTO.2001.91.10.924

[11] Tang, S.Y., Xiao, Y.N. and Cheke, R.A. (2010) Dynamical Analysis of Plant Disease Models with Cultural Control Strategies and Economic Thresholds. Mathematics and Computers in Simulation, 80, 894-921. https://doi.org/10.1016/j.matcom.2009.10.004

[12] Gao, S.J., Zhang, L.J., Xie, D. and Zhang, F. (2016) Varying Pulse Control Schemes for Citrus Huanglongbing Epidemic Model with General Incidence. Communications in Mathematical Biology and Neuroscience, 2016, 7.

[13] Gao, S.J., Chen, L.S. and Teng, Z.D. (2007) Impulsive Vaccination of an SEIRS Model with Time Delay and Varying Total Population Size. Bulletin of Mathematical Biology, 69, 731-745. https://doi.org/10.1007/s11538-006-9149-x

[14] Wang, W. and Zhao, X.-Q. (2008) Threshold Dynamics for Compartmental Epidemic Models in Periodic Environments. Journal of Dynamics and Differential Equations, 20, 699-717. https://doi.org/10.1007/s10884-008-9111-8

[15] Yang Y.P., and Xiao, Y.N. (2012) Threshold Dynamics for Compartmental Epidemic Models with Impulses. Nonlinear Analysis. Real World Applications, 13, 224-234. https://doi.org/10.1016/j.nonrwa.2011.07.028

[16] Wang, J.P., Feng, F.F., Guo, Z., Lv, H. and Wang, J.J. (2019) Threshold Dynamics of a Vector-Borne Epidemic Model for Huanglongbing with Impulsive Control. Applied Mathematics, 10, 196-211. https://doi.org/10.4236/am.2019.104015

[17] Bainov, D. and Simeonov, P.S. (1993) Impulsive Differential Equations: Periodic Solutions and Applications. Longman Harlow, New York.

[18] Zhang, T.Q., Meng, X.Z., Song, Y. and Li, Z. (2012) Dynamical Analysis of Delayed Plant Disease Models with Continuous or Impulsive Cultural Control Strategies. Abstract and Applied Analysis, 2012, Article ID: 428453. 
https://doi.org/10.1155/2012/428453

[19] Teng, Z. and Wang, L. (2016) Persistence and Extinction for a Class of Stochastic SIS Epidemic Models with Nonlinear Incidence Rate. Physica A, 451, 507-518. https://doi.org/10.1016/j.physa.2016.01.084

[20] Pei, Y.Z., Ji, X.H. and Li, C.G. (2010) Pest Regulation by Means of Continuous and Impulsive Nonlinear Controls. Mathematical and Computer Modelling, 51, 810-822. https://doi.org/10.1016/j.mcm.2009.10.013 